\section{Malnutrition as a prognostic factor in lymphoblastic leukaemia: a multivariate analysis}

EDITOR,-Borato Viana et al report evidence that malnutrition is an adverse prognostic factor in childhood acute lymphoblastic leukaemia (ALL), suggest that the effect may apply even to moderately undernourished children, and discuss some possible mechanisms. ${ }^{1}$ While the relevance of severe malnutrition to the developed world may seem limited, our own evidence ${ }^{2}$ indicates that relatively mild undernutrition (weight for height SD score $<-0.5)$ had adverse prognostic significance for children treated for ALL in Glasgow on the UKALL-X protocol. We would also like to add two possible mechanisms which may be worthy of investigation: impairment of immune function by undernutrition and variability in body composition between patients producing variation in drug pharmacokinetics. ${ }^{3}$

There are now three studies which indicate the need for further research on the relevance of nutritional status to outcome in ALL, and on the possible mechanisms. ${ }^{124}$

JOHN J REILLY
I ODAME
B E GIBSON
B A WHARTON
University of Glasgow,
Department of Human Nutrition
and Department of Haematology,
Royal Hospital for Sick Children,
Yorkhill,
Glasgow G3 $8 S f$

1 Borato Viana M, Murao M, Ramos G, et al. Malnutrition as a prognostic factor in lymphoblastic leukaemia. a multivariate analysis. Arch Dis Child 1994; 71: 304-10.

2 Reilly JJ, Odame I, McColl JH, McAllister PJ, Gibson BES, Wharton BA. Does weight Gibson BES, Wharton BA. Does weigh for height have prognostic significance in children with acute lymphoblastic leukaemia? Am ${ }_{225}$-30.

3 Reilly JJ, Workman P. Is body composition an important variable in the pharmacokinetics of anticancer drugs? Cancer Chemother Pharmacol 1994; 34: 3-13.

4 Lobato-Mendizabal E, Ruis-Arguelles GJ, MariaLopez A. Leukaemia and nutrition I: malnutrition is an adverse prognostic factor in the outcome of treatment of patients with standard risk acute lymphoblastic leukaemia. Leuk Res 1989; 13: 899-906.

\section{Central nervous system tumours - lack of national studies}

EDITOR,-Drs Thorne and Foreman, in their letter published in July, point out that children with brain tumours have not been allowed the advantage of participating in national trials because of the lack of such studies and exhort the Medical Research Council (MRC) and UK Children's Cancer Study Group (UKCCSG) to address this issue. 1

Their letter unfortunately ignores the fact that the UKCCSG has been working with the International Society of Paediatric Oncology to run clinical trials for children with primitive neuroectodermal tumours since the mid 1970 s. The major problem faced by both the UKCCSG and the International Society for Paediatric Oncology has been a reluctance by the neurosurgical community, to whom most of these patients present in the first instance, to pass their clinical care onto paediatric oncologists. Indeed, at a time when $80 \%$ of children with malignant disease are being referred to paediatric oncology centres, only
$46 \%$ of children with central nervous system tumours are being so referred.

The group has recently expanded its area of activities, studies are currently open for the treatment of children with primitive neuroectodermal tumour, brain stem glioma, and for the treatment of infants under the age of 3 years. In the near future, protocols will open for the treatment of children with low and high grade astrocytomas and for intracranial germ cell tumours.

The problem therefore lies not with the efforts of the UKCCSG or the MRC to promote such trials, but with the cooperation that we need to receive from our neurosurgical colleagues, and a willingness from the paediatric oncology community to enter these children to randomised clinical studies. CLIFFORD C BAILEY
Yorkshire Regional Centre Paediatric Oncology and St fames's University Hospital, Leeds LS9 7TF

1 Thorne R, Foreman NK. Central nervous system tumours lack national studies. Arch Dis Child 1994; 71: 95-6.

\section{Audit of neonatal intensive care transport}

EDITOR,-Two articles concerning transpor of the critically ill neonate and child appear in the July issue of the journal. ${ }^{12}$ Seventy five per cent of 56 children transported had adverse clinical events. ${ }^{2}$ This is an extremely high rate. A report of complications during transport of 614 patients in North Carolina reported complications in the neonate of $3.1 \%$ and $1.5 \%$ in paediatric patients less than 1 year old. Beyond 1 year no complications were observed. ${ }^{3}$ I agree strongly with the authors ${ }^{2}$ that inadequate stabilisation before transportation is the principal cause of complications during transfer. We have documented the time taken for stabilisation in 2863 neonatal and paediatric patients. ${ }^{4}$ The median stabilisation time for a neonate was $\mathbf{8 0}$ minutes and for a paediatric patient was 45 minutes. The time for stabilisation of neonate reported by the Nottingham group of 75 minutes is comparable with our experience. ${ }^{4}$ This is time very well spent. Unfortunately, a common but deplorable approach to transport reflecting an attitude of 'get that patient out of here' is very common and detrimental to patient safety during transport. This attitude of so called 'swoop and scoop' with inadequate time spent on stabilisation is rarely if ever appropriate in interfacility transfer of sick patients. There are those who claim 75-80 minutes spent stabilising a sick newborn before transfer is unnecessary - the so called (and cynical) 'stay and play' philosophy. I disagree with this. Rather stay and play and be assured a thorough stabilisation than swoop and scoop with serious but avoidable problems during the transfer.

An in-depth, very practical, and helpful revision of the 1986 American Academy of Pediatric guidelines for air and ground transportation of paediatric patients had been recently published ${ }^{5}$ and is highly recommended for all facilities engaging in paediatric and neonatal transport.

$$
\begin{array}{r}
\text { JONATHAN M WHITFIELD } \\
\text { Neonatology, } \\
\text { Baylor College of Medicine, } \\
1 \text { Baylor Plaza, } \\
\text { Houston, } \\
\text { Texas } 77030 \text {, USA }
\end{array}
$$

1 Leslie AJ, Stephenson TJ. Audit of neonatal intensive care transport. Arch Dis Child 1994; 71: F61-6.

2 Barry PW, Ralston C. Adverse events occurring during interhospital transfer of the critically ill Arch Dis Child 1994; 71: 8-11.

3 Beyer AJ III, Land G, Zaritsky A. Nonphysician transport of intubated pediatric patients: a system evaluation. Crit Care Med 1992; 20: 961-6.

4 Whitfield JM, Buser MK. Transport stabilization times for neonatal and pediatric patients prio to interfacility transfer. Pediatr Emerg Care 1993; 9: 69-71

5 McCloskey K, Hackel A. Guidelines for air and ground transport of neonatal and pediatric patients. Pediatrics, 1993.

\section{Familial occurrence of congenital} laryngeal clefts

EDrTOR,-In your journal in 1973, in association with the late J G Stocks, we reported two sibships of double first cousins in which six children had congenital laryngeal anomalies. ${ }^{1}$ Three of the children had proved congenital posterior laryngeal clefts, one had subglottic stenosis with a deformed cricoid cartilage, and it is likely that the other two had posterior laryngeal clefts.

Although these occurred in only one generation, we speculated that the mode of inheritance may well have been autosomal dominant. We relied primarily on statistical argument that dominant inheritance was more likely as there were only three unaffected children in the two sibships. Five siblings of the mothers had died in infancy and the sixth had stridor all his life which we took as support for the suggested mode of inheritance.

We have now seen the first child of patient 16 (Lyndal) in that report. He was diagnosed at laryngoscopy and bronchoscopy as having a posterior laryngeal cleft which extended down to the circoid cartilage. The father of the child was unrelated to the mother. Patient 14 (Judith) has two children, one of whom has laryngomalacia but no cleft and the other has no symptoms of laryngeal disease. The other affected women have no children.

The occurrence of this malformation in the next generation of the sibships we reported supports our hypothesis that in this family congenital laryngeal clefts are inherited as autosomal dominant trait.

PETER D PHELAN
HOWWRD WILIIAMS
Department of Paediatrics (University of Melbourne),
Royal Children's Hospital,
Parkville,
Victoria 3052,
Australia
DAVID DANKS
Murdoch Institute,
Melbourne,
Australia

\section{Female genital mutilation}

EDITOR,-Why do we call it female genital mutilation, when a similar barbaric operation carried out for equally arcane tribal reasons on male genitalia is called circumcision, and doesn't seem to worry the child abuse specialists?

Shouldn't both be banned, both allowed, or examples of both practices referred to 